\title{
ART-XC Instrument on board of the SRG Mission
}

M. Pavlinsky ${ }^{1}$, V. Akimov ${ }^{1}$, V. Levin ${ }^{1}$, I. Lapshov ${ }^{1}$, A. Tkachenko ${ }^{1}$, N. Semena ${ }^{1}$, V. Arefev ${ }^{1}$, A.Glushenko ${ }^{1}$, A. Yaskovich ${ }^{1}$, R. Burenin ${ }^{1}$, S. Sazonov ${ }^{1}$, M. Revnivtsev ${ }^{1}$, M. Buntov ${ }^{1}$, S. Grebenev ${ }^{1}$, A.Lutovinov'1, M. Kudelin¹, S. Grigorovich², D. Litvin², V. Lazarchuk2, I. Roiz², M. Garin',

M.Gubarev³, B.Ramsey³, K. Kilaru³, S.L. O"Dell, R. Elsner³

1 - Space Research Institute (Russian Federation)

2 - The All-Russian Scientific Research Institute for Experimental Physics, VNIIEF (Russian Federation)), 3 - NASA Marshall Space Flight Ctr., USA 


\section{Spectrum-R-Gamma Scientific Payload}

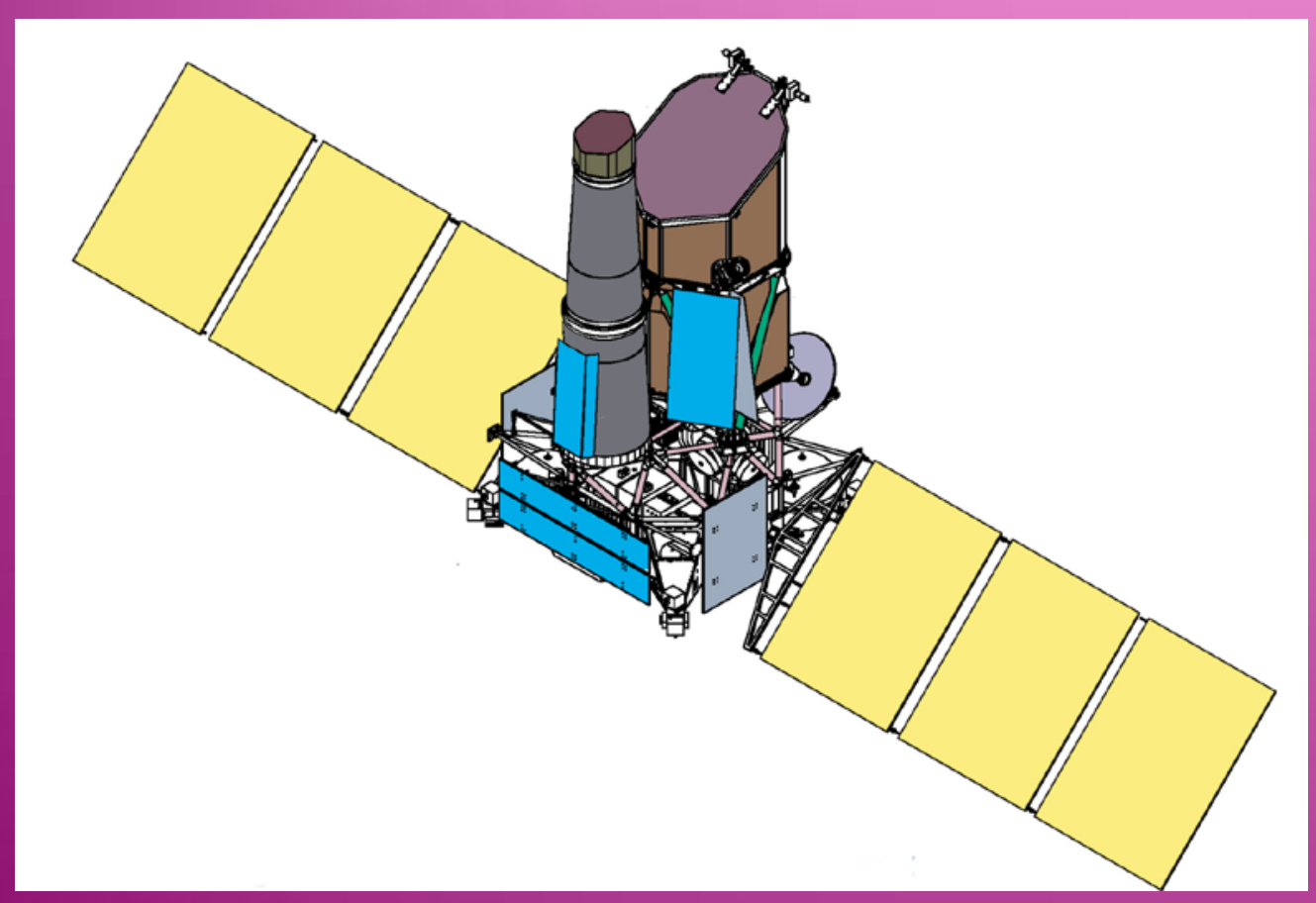

eROSITA - extended ROentgen Survey with an Imaging Telescope Array (MPE, Germany), 7 mirror systems

Energy range $0.2-12.0 \mathrm{keV}$,

FOV $\sim 1^{\circ}$, PSF $\sim 20^{\prime \prime}$ (FOV averaged) and $\sim 15^{\prime \prime}$ on axis,

Energy resolution $130 \mathrm{eV}$ at $6 \mathrm{keV}$, effective area $2500 \mathrm{~cm}^{2}$, Grasp of $\sim 700 \mathrm{~cm}^{2} \mathrm{deg}^{2}$ at $1 \mathrm{keV}$;

ART-XC - Astronomical oentgen Telescope - X-ray oncentrator (IKI, Russia), 7 mirror systems.

Energy range 6 - $30 \mathrm{keV}$,

FOV $\sim 32$ ', energy resolution $\sim 10 \% \mathrm{keV}$ at $14 \mathrm{keV}$,

Effective area $\sim 510 \mathrm{~cm}^{2}$ at $7 \mathrm{keV}$, a Grasp of $\sim 45 \mathrm{~cm}^{2} \mathrm{deg}^{2}$ at $7 \mathrm{keV}$. 


\section{Launcher, buster, platform, orbit and observation strategy of the SRG Mission}

- The Spectrum-Roentgen-Gamma mission will be launched with the Zenit launcher and the Fregat buster from Baikonur in September 2013

- SRG utilizes the newly-developed Navigator platform

- The SRG observatory will be launched to $L 2$ point.

- The SRG observing program is divided into three stages over the 7-year mission lifetime.

- The first 3-month during the transit to the L2 point, will be devoted to initial check-up and in-flight calibration.

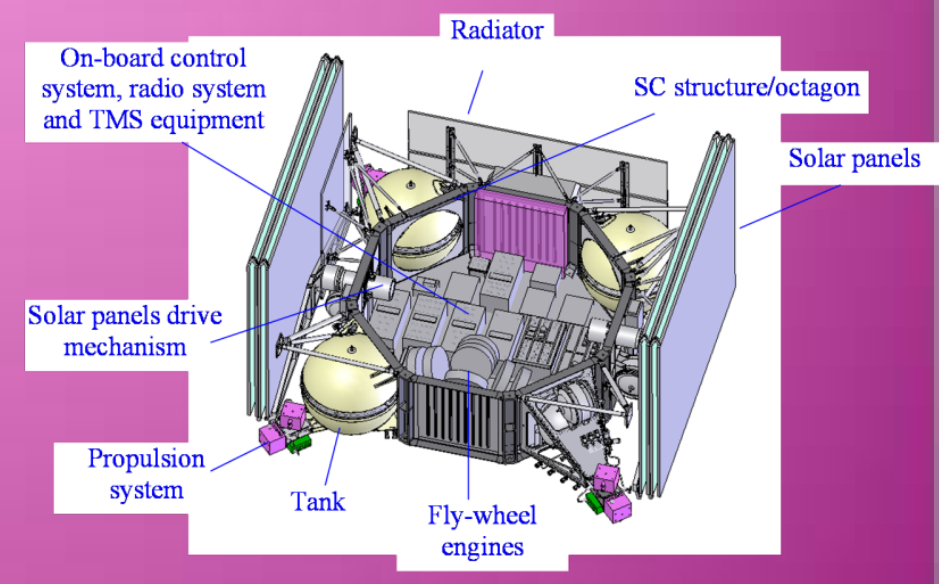

The Navigator platform

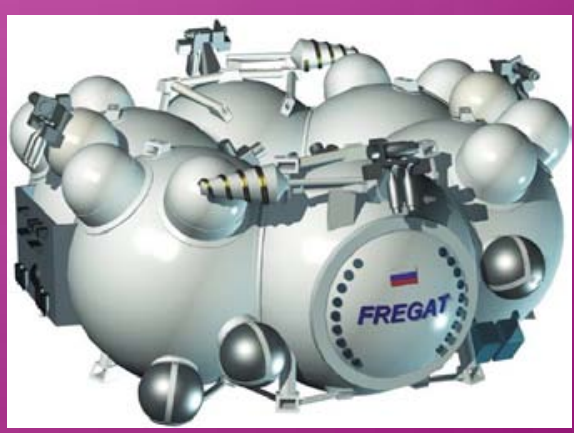

The Fregat booster 


\section{ART-XC Scientific goals}

All-sky $X$-ray survey at $6-11$ keV energy region with sensitivity $\leq 3 \times 10^{-13} \mathrm{erg} \mathrm{s}^{-1} \mathrm{~cm}^{-2} \mathrm{keV}^{-1}$, discovery in course of survey at local Universe several thousands new active galaxies nucleus (AGNs);

Study of intrinsically heavily absorbed/Compton thick AGNs $\left(N_{H} \geq\right.$ $3 \times 10^{23} \mathrm{CM}^{-2}$ );

Study of massive nearby galaxies clusters with $\mathrm{T} \geq 4 \mathrm{keV}$ in pointing observation mode;

Study heavily obscured galactic X-ray binary systems;

Study broad band spectra of Galactic objects (including binary systems, anomalous pulsars, supernova remnants) up to $30 \mathrm{keV}$, spectroscopy and timing of point sources;

Study non-thermal component in the Galaxy diffuse emission.

Search for cyclotron lines features $X$-ray pulsars spectra 


\section{ART-XC general design}

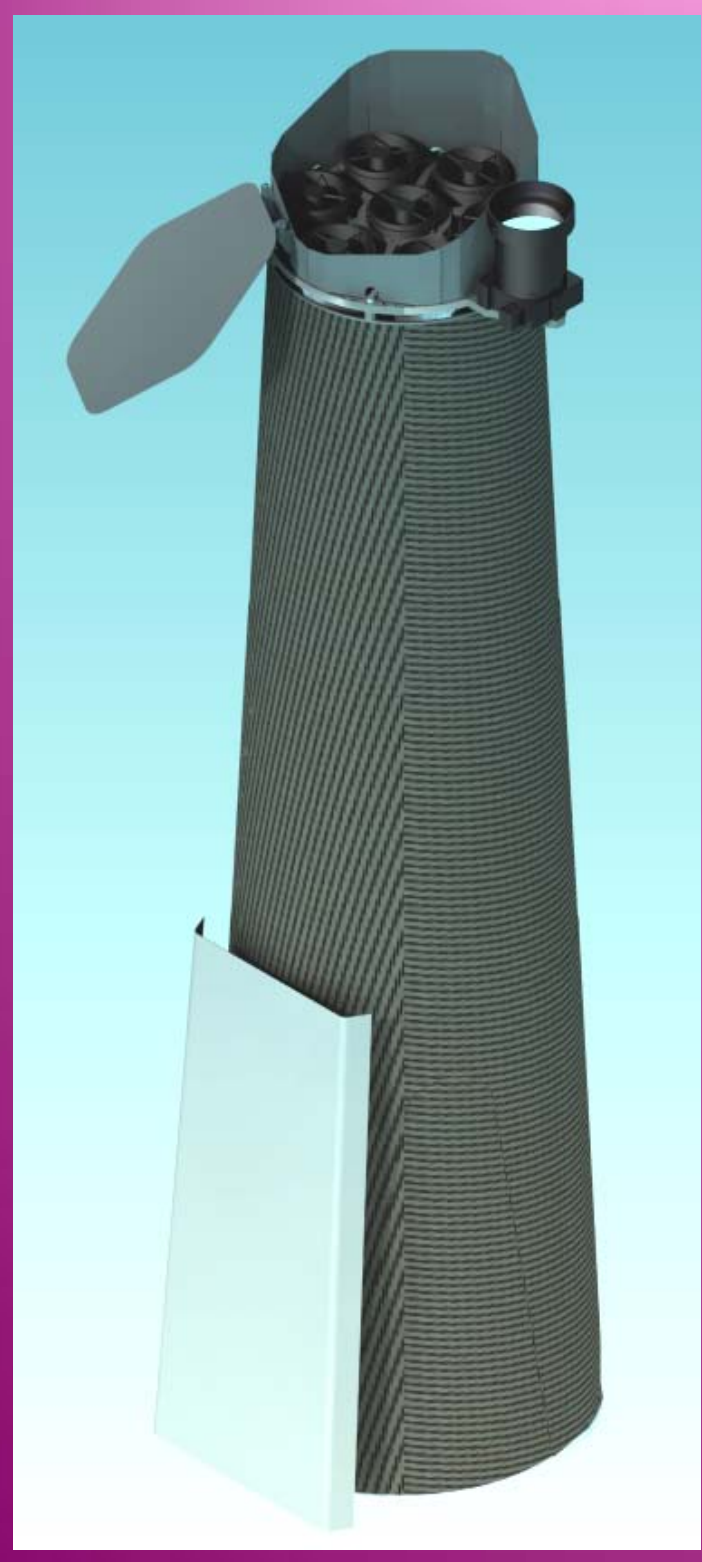

\begin{tabular}{|c|c|}
\hline Number of mirror systems & 7 \\
\hline Number of nested mirror shells & 28 \\
\hline $\begin{array}{l}\text { Mirror shells and coating } \\
\text { materials }\end{array}$ & Nickel and Iridium \\
\hline Focal length & $2700 \mathrm{~mm}$ \\
\hline FOV & $\varnothing 32^{\prime}$ \\
\hline Angular resolution & $<1^{\prime}$ \\
\hline $\begin{array}{l}\text { Effective Area for Pointed } \\
\text { Observations }\end{array}$ & 510 cm²@ $@ 7$ keV \\
\hline Grasp for Survey & $\begin{array}{l}45 \mathrm{deg}^{2} \mathrm{~cm}^{2} @ \\
7 \mathrm{keV}\end{array}$ \\
\hline Detector type & DSSD CdTe \\
\hline Size & $25.6 \times 25.6 \mathrm{~mm}^{2}$ \\
\hline Number of strips & $64 \times 64$ \\
\hline Strip pitch & $0.4 \mathrm{~mm}$ \\
\hline Energy range & $6-30 \mathrm{keV}$ \\
\hline Energy resolution & $10 \%$ at $14 \mathrm{keV}$ \\
\hline Time resolution & $1 \mathrm{~ms}$ \\
\hline
\end{tabular}

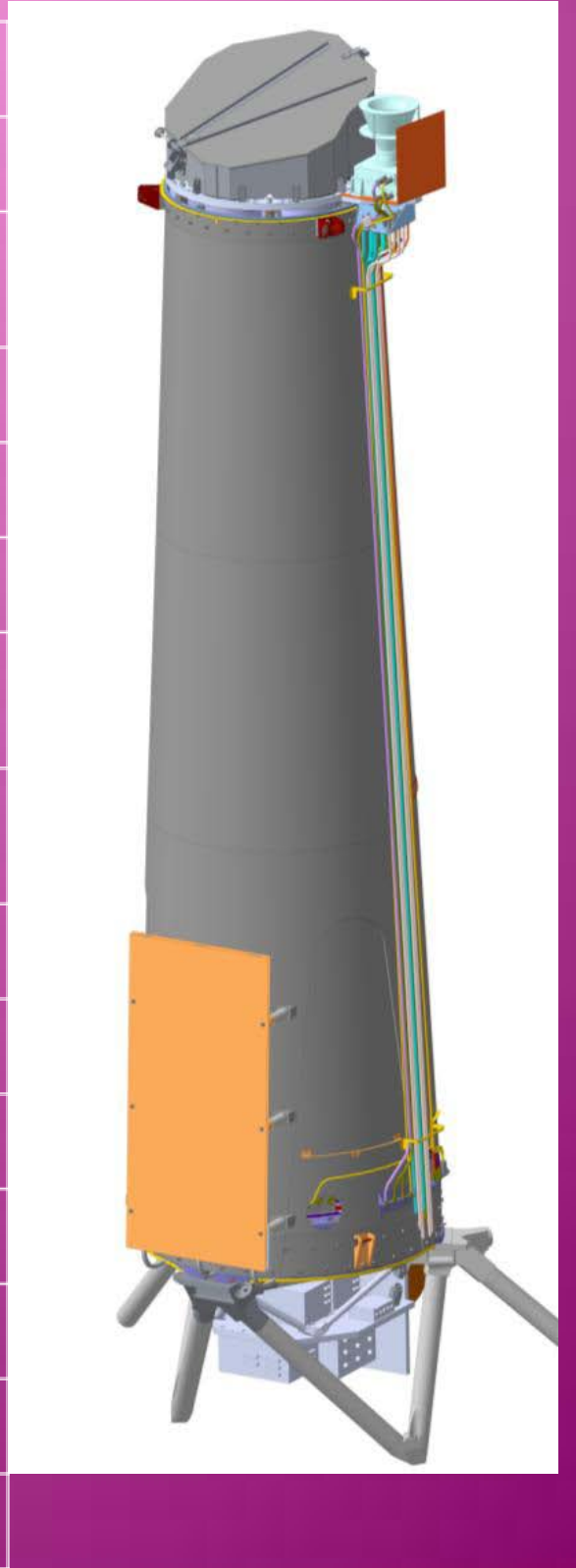




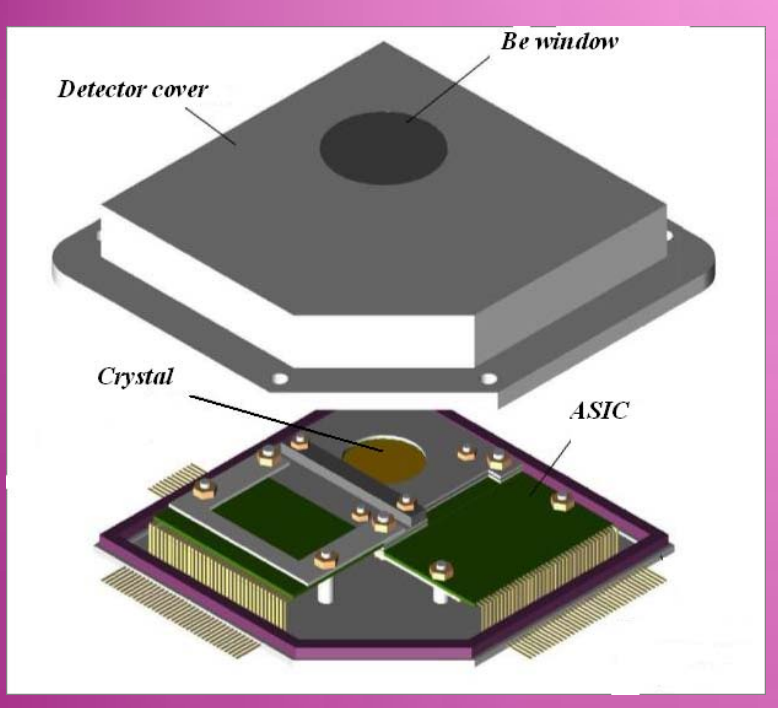

Schematic of the CdTe detector in vacuum tight internal box

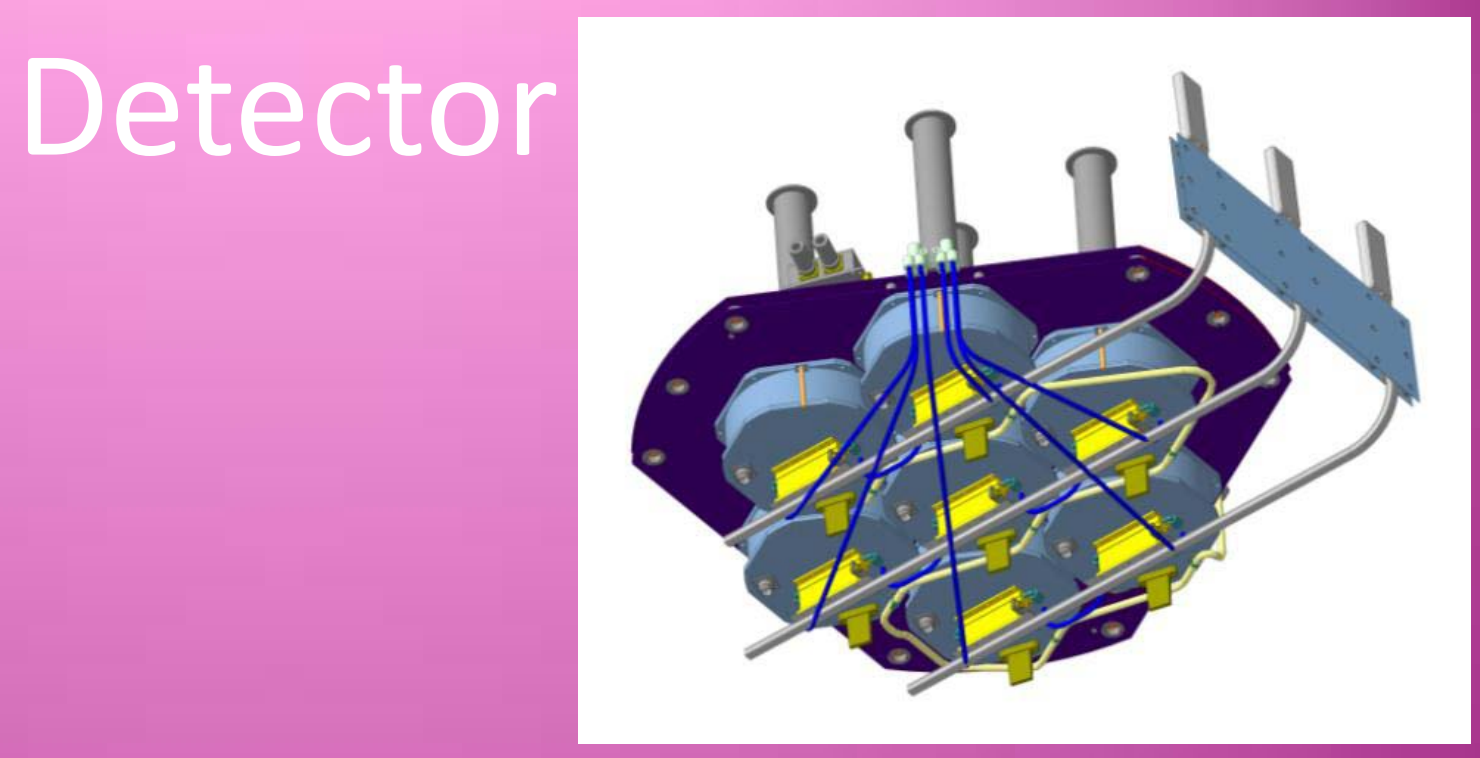

Seven detectors with pre-collimators and passive cooling system installed at the focal plane of the ART_XC telescope.

\begin{tabular}{|c|c|}
\hline Detector type & CdTe Schottky Diode double sided strip (ACRORAD) \\
\hline Crystal size & $30 \times 30 \times 1$ мM \\
\hline Strip width & 550 micron \\
\hline Inter-strip distance & 75 micron \\
\hline Number of strips & $41 \times 41$ \\
\hline ASIC & VA64TA \\
\hline Energy range & 6 -30 keV \\
\hline Energy resolution & $10 \%$ at $14 \mathrm{keV}$ \\
\hline Operating temperature & -30 to -40 degrees C \\
\hline Be window thickness & 100 micron \\
\hline
\end{tabular}




\section{X-ray Optics}

- The X-ray modules are fabricated by the VNIIEF (Russia) and MSFC (USA);

- The NASA-IKI Reimbursable Agreement has been signed on February 72011 to build 4 flight units;

- NASA is to deliver 4 flight modules for the ART-XC instruments by June 24, 2013;

- The work at the MSFC has been started on March 242011. 


\section{Requirements}

Optical Performance Requirements:

\section{Encircled Half Energy Width}

- On axis Less than $1 \mathrm{~mm}$ diameter

- 15'off axis Less than $2.5 \mathrm{~mm}$ diameter

Mirror Module Effective Area $\geq 65 \mathrm{~cm}^{2}$ at $8 \mathrm{keV}$ (on axis)

Weight Less than $17 \mathrm{~kg}$

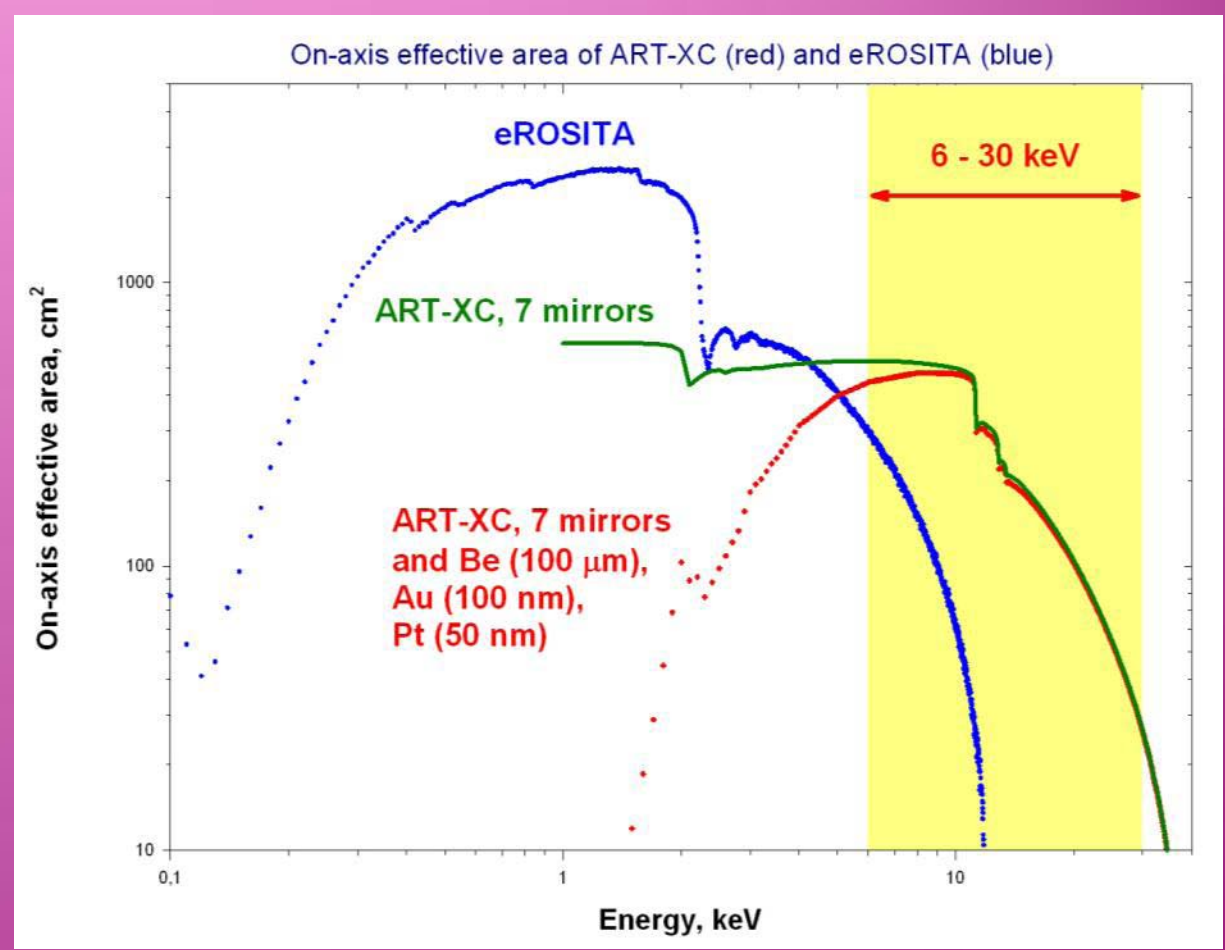




\section{X-ray optics}

Both the VNIIEF and the MSFC designs are based on the single spider scheme

$>$ The shell diameters vary from 50 to $150 \mathrm{~mm}$

$>$ The VNIIEF x-ray module design calls for the mirror shell thickness of 250 micron. The vibration tests for the qualification unit are in progress;

$>$ The MSFC is exploring the variable thickness option. The design of the ART spider and housing is in progress.

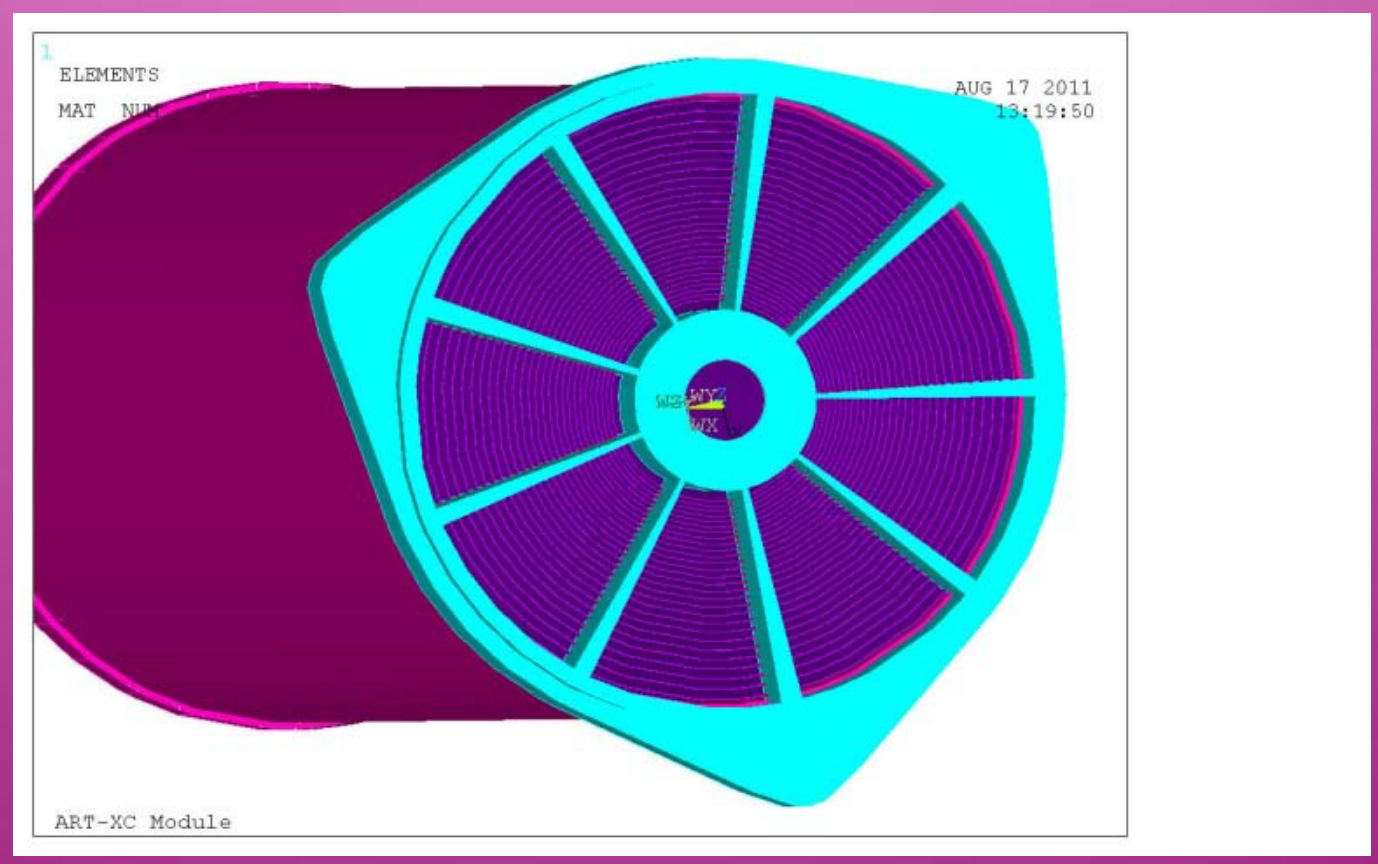

A model view of the current MSFC concept, which will be very close to the final design. The spider is holding 28 mirror shells. The protective cover is also installed on the spider 


\section{Optical performance}
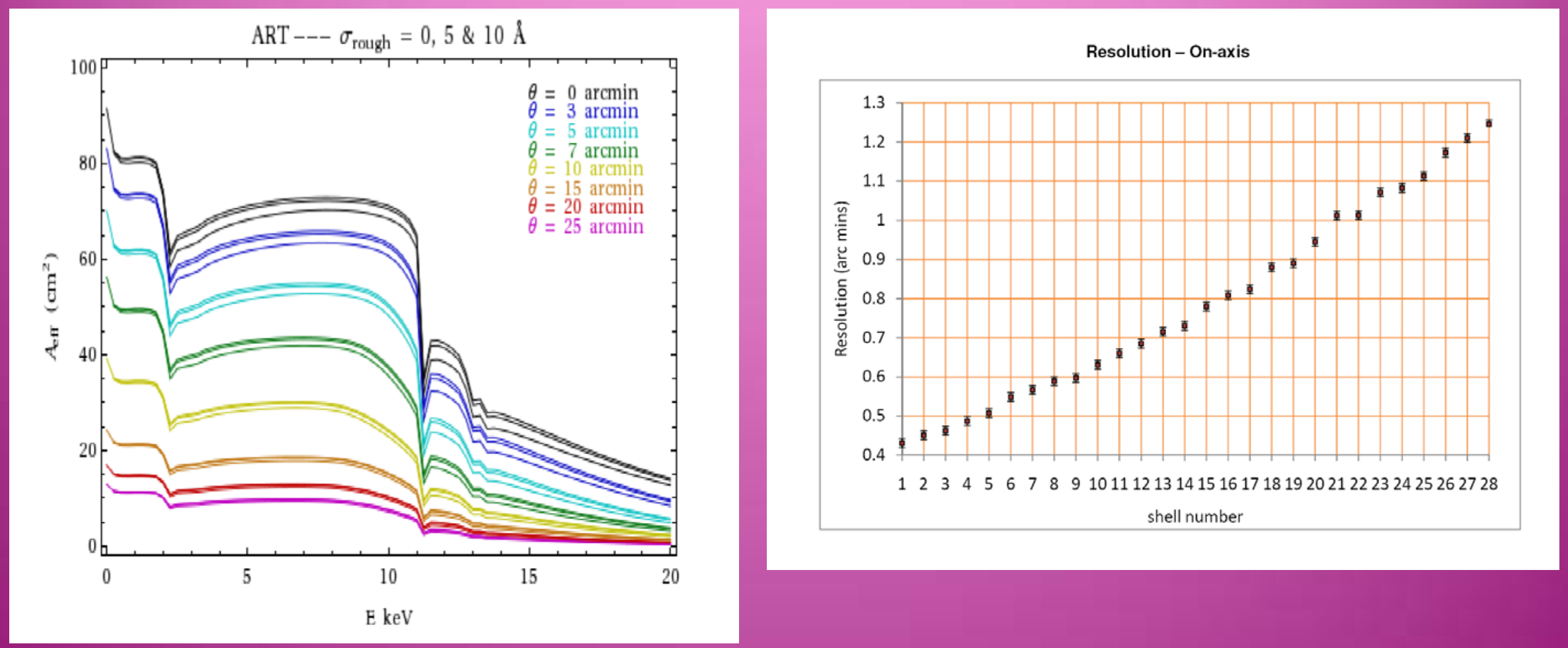

To meet the optical angular resolution requirements the shell mirrors need to be fabricated to the Walter prescription.

To meet the effective area requirements the surface roughness of the mirrors has to be below $6 \mathrm{~A}$. 


\section{Summary of current status}

- The SRG Mission is scheduled to be launched in September 2013

- The optics for the ART-XC Instrument to be build by the VNIIEF (Russia) and the MSFC (USA)

- The mandrel fabrication has been started 